\title{
COMPETITION IN THE GRADOSTAT FOR THE CASE OF THREE VESSELS AND THREE POPULATIONS
}

\author{
H. EL-OWAIDY, A.A. AMMAR AND O.A. EL-LEITHY
}

\section{Introduction}

Continuous culture of microorganisms using the chemostat is an important technique in microbiology and population biology used to study properties of population growth and interactions among microorganisms. The chemostat is an ecosystem model,representing a simple lake, where competition can be studied experimentally [3], computationally [13], and analytically [4], [6], [7]. In its simplest form, the chemostat, consists of several populations of microorganisms competing for a single limiting nutrient.

Substrate concentration in many ecosystems is however, not homogeneous, and in many cases it occurs in gradients. To study such microbial ecosystems, Lovitt and Wimpenny [9], [15] devised a concentration of chemostats-called a gradostat-consisting of a series of chemostats in which the adjacent vessels are linked together.

The competition in the gradostat has been analyzed in [8], for the case of two vessels and two populations. In this paper, we extend such analysis for the case of three vessels and three populations. The mathematical arguments presented are a combination of computational ones and geometric ones.

The model is described as a system of twelve nonlinear differential equations in Section 2. The omega limit set lies in a nine dimensional set, so it is sufficient to work there. The set of equilibrium (also closed critical points, rest points or steady and these names are used interchangeably in this paper) of this system, in Section 3. In section 4 we investigate the steady state, first, with one population, since it is necessary to study the flow on the boundary. Simple geometric arguments are used. In section 5 we will investigate the stability of the rest points. The size of the variational matrix, $9 \times 9$, makes the computation difficult.

\section{The Model}

The system we will analyze consists of three chemostats, such that the adjacent vessels are connected. The derivation for chemostats and the general gradostat can be found in many literatures such as [4], [9], [14], [15]. The basic property is that transport rates and the volumes are constant, so the rate of transports between contiguous vessels, as

Received March 22, 1989. 
well as, the flow rates out of each vessel are all the same. Moreover, in order to keep the volume constant, one assumes the addition of mediumwithout the limiting nutrient-in the right hand vessel.

For each $i, i=1,2,3$; let $S_{i}(t), u_{i}(t), v_{i}(t), \ell_{i}(t)$, be respectively the concentration of the substrate (nutrient) and of the three populations of competing microorganisms in the $i$ th vessel at time $t$, the model equations are:

$$
\begin{aligned}
& s_{i}^{\prime}=\left(s_{i-1}-2 s_{i}+s_{i+1}\right) D-f_{u}\left(s_{i}\right) u_{i} / y_{u}-f_{v}\left(s_{i}\right) v_{i} / y_{v}-f_{\ell}\left(s_{i}\right) \ell_{i} / y_{\ell} \\
& u_{i}^{\prime}=\left(u_{i-1}-2 u_{i}+u_{i+1}\right) D+f_{u}\left(s_{i}\right) u_{i}, \\
& v_{i}^{\prime}=\left(v_{i-1}-2 v_{i}+v_{i+1}\right) D+f_{v}\left(s_{i}\right) v_{i}, \\
& \ell_{i}^{\prime}=\left(\ell_{i-1}-2 \ell_{i}+\ell_{i+1}\right) D+f_{\ell}\left(s_{i}\right) \ell_{i}, . \\
& s_{0}=s^{0}, s_{4}=u_{4}=v_{4}=\ell_{4}=0, u_{0}=v_{0}=\ell_{0}=0, \\
& s_{i}(0) \geq 0, u_{i}(0) \geq 0, v_{i}(0) \geq 0, \ell_{i}(0) \geq 0 ; i=1,2,3 .
\end{aligned}
$$

The functions

$$
f_{u}\left(s_{i}\right)=\frac{m_{u s} s_{i}}{a_{u}+s_{i}}, f_{v}\left(s_{i}\right)=\frac{m_{v} s_{i}}{a_{v}+s_{i}}, \text { and } f_{\ell}\left(s_{i}\right)=\frac{m_{\ell} s_{i}}{a_{\ell}+s_{i}}, i=1,2,3 \text {, }
$$

are the usual Michaelis-Menten response terms. $s^{0}$ is the constant input concentration of the limiting nutrient. $D$ is the constant washout rate. $a_{u}, a_{v}, a_{\ell}$ are positive constants which are the Michaelis-Menten constants for the $u, v, \ell$ populations while the constants $m_{u}, m_{v}, m_{\ell}$ are the corresponding maximal growth rates, and $y_{u}, y_{v}, y_{\ell}$ are the corresponding yield constants.

The variables in the above system may rescaled by measuring $s, u, v$ and $\ell$, respectively, in units of $s^{0}, s^{0} y_{r}, s^{0} y_{v}$ and $s^{0} y_{\ell}$. Moreover, $D$ may be scaled out. The system takes the form:

$$
\begin{aligned}
& s_{1}^{\prime}=1-2 s_{1}+s_{2}-f_{u}\left(s_{1}\right) u_{1}-f_{v}\left(s_{1}\right) v_{1}-f_{\ell}\left(s_{1}\right) \ell_{1}, \\
& s_{2}^{\prime}=s_{1}-2 s_{2}+s_{3}-f_{u}\left(s_{2}\right) u_{2}-f_{v}\left(s_{2}\right) v_{2}-f_{\ell}\left(s_{2}\right) \ell_{2}, \\
& s_{3}^{\prime}=s_{2}-2 s_{3}-f_{u}\left(s_{3}\right) u_{3}-f_{v}\left(s_{3}\right) v_{3}-f_{\ell}\left(s_{3}\right) \ell_{3}, \\
& u_{1}^{\prime}=-2 u_{1}+u_{2}+f_{u}\left(s_{1}\right) u_{1}, \\
& u_{2}^{\prime}=u_{1}-2 u_{2}+u_{3}+f_{u}\left(s_{2}\right) u_{2}, \\
& u_{3}^{\prime}=u_{2}-2 u_{3}+f_{u}\left(s_{3}\right) u_{3} \\
& v_{1}^{\prime}=-2 v_{1}+v_{2}+f_{v}\left(s_{1}\right) v_{1} \\
& v_{2}^{\prime}=v_{1}-2 v_{2}+v_{3}+f_{v}\left(s_{2}\right) v_{2}, \\
& v_{3}^{\prime}=v_{2}-2 v_{3}+f_{v}\left(s_{3}\right) v_{3} \\
& \ell_{1}^{\prime}=-2 \ell_{1}+\ell_{2}+f_{\ell}\left(s_{1}\right) \ell_{1} \\
& \ell_{2}^{\prime}=\ell_{1}-2 \ell_{2}+\ell_{3}+f_{\ell}\left(s_{2}\right) \ell_{2}, \\
& \ell_{3}^{\prime}=\ell_{2}-2 \ell_{3}+f_{\ell}\left(s_{3}\right) \ell_{3}, \\
& s_{i}(0) \geq 0, u_{i}(0) \geq 0, v_{i}(0) \geq 0, \ell_{i}(0) \geq 0 ; i=1,2,3 .
\end{aligned}
$$


It is clear that the $a$ 's and $m$ 's have changed their biological meaning although the new $f$ 's have the same form as those in (2.1). We analyze the system (2.2). Let $R_{+}^{n}$ be the closed nonnegative cone.

$$
R_{+}^{n}=\left\{x \in R^{n}: x_{i} \geq 0, i=1, \ldots, n\right\}
$$

and let

$$
R_{+}^{o n}=\left\{x \in R^{n}: x_{i}>0, i=1, \ldots, n\right\}
$$

be the open positive cone.

Iemma 2.1 (i) $R_{+}^{12}$ is positively invariant under the solution map of (2.2).

(ii) Solutions of (2.2) are uniformly asymptotically bounded as $t \rightarrow \infty$, i.e. the system (2.2) is dissipative. In fact,

$$
\begin{aligned}
& \lim _{t \rightarrow \infty} s_{1}(t)+u_{1}(t)+v_{1}(t)+\ell_{1}(t)=3 / 4 \\
& \lim _{t \rightarrow \infty} s_{2}(t)+u_{2}(t)+v_{2}(t)+\ell_{2}(t)=1 / 2 \\
& \lim _{t \rightarrow \infty} s_{3}(t)+u_{3}(t)+v_{3}(t)+\ell_{3}(t)=1 / 4 .
\end{aligned}
$$

Moreover, the set:

$$
\begin{aligned}
\Gamma= & \left\{\left(s_{1}, s_{2}, s_{3}, u_{1}, u_{2}, u_{3}, v_{1}, v_{2}, v_{3}, \ell_{1}, \ell_{2}, \ell_{3}\right)\right. \\
& \left.\in R_{+}^{12} \mid s_{1}+u_{1}+v_{1}+\ell_{1}=3 / 4, s_{2}+u_{2}+v_{2}+\ell_{2}=1 / 2, s_{3}+u_{3}+v_{3}+\ell_{3}=1 / 4\right\}
\end{aligned}
$$

is positively invariant.

Proof. (i) and (ii) since the argument are quite standard, we omit the details, save to note that if $z_{i}(t)=s_{i}(t)+u_{i}(t)+v_{i}(t)+\ell_{i}(t) ; i=1,2,3$; then

$$
\begin{aligned}
z_{1}^{\prime} & =1-2 z_{1}+z_{2} \\
z_{2}^{\prime} & =z_{1}-2 z_{2}+z_{3} \\
z_{3}^{\prime} & =z_{2}-2 z_{3}
\end{aligned}
$$

Rewrite the equation (2.3) in vector form:

$$
z^{\prime}=A Z+e, \quad z(0) \geq 0
$$

where

$$
A=\left(\begin{array}{ccc}
-2 & 1 & 0 \\
1 & -2 & 1 \\
0 & 1 & -2
\end{array}\right), z=\left(\begin{array}{l}
z_{1} \\
z_{2} \\
z_{3}
\end{array}\right), e=\left(\begin{array}{l}
1 \\
0 \\
0
\end{array}\right)
$$

The eigenvalues of $A$ are:

$$
\lambda_{1}=-2, \quad \lambda_{2,3}=-2 \pm \sqrt{2} .
$$

Since all the eigenvalues are negative, the solution $z(t)$ of the initial value problem is bounded and asymptotically approach the steady state, $-A^{-1} e$, and so the result follows. 
Iemma 2.1 Suggests that the omega limit set of any trajectory is nonempty, compact, connected and contained in $\Gamma$. We look for first to analyze the flow on the omega limit set of (2.2) because every trajectory asymptotic to its omega limit set. The trajectories in the omega limit set satisfy

$$
\begin{aligned}
& u_{i}^{\prime}=u_{i-1}-2 u_{i}+u_{i+1}+f_{u}\left(\left(1-\frac{i}{4}\right)-u_{i}-v_{i}-\ell_{i}\right) u_{i}, \\
& v_{i}^{\prime}=v_{i-1}-2 v_{i}+v_{i+1}+f_{v}\left(\left(1-\frac{i}{4}\right)-u_{i}-v_{i}-\ell_{i}\right) v_{i}, \\
& \ell_{i}^{\prime}=\ell_{i-1}-2 \ell_{i}+\ell_{i+1}+f_{\ell}\left(\left(1-\frac{i}{4}\right)-u_{i}-v_{i}-\ell_{i}\right) \ell_{i}, \\
& u_{0}=v_{0}=\ell_{0}=0=u_{4}=v_{4}=\ell_{4}, \\
& u_{i}(0) \geq 0, v_{i}(0) \geq 0, \ell_{i}(0) \geq 0, i=1,2,3 ; \\
& \frac{3}{4}-u_{1}-v_{1}-\ell_{1} \geq 0, \frac{1}{2}-u_{2}-v_{2}-\ell_{2} \geq 0 \text { and } \frac{1}{4}-u_{3}-v_{3}-\ell_{3} \geq 0 .
\end{aligned}
$$

\section{The Critical Set}

We will first search for the equilibrium points for the full system (2.2) and deduce what is needed for (2.2).

Lemma 3.1 Let $\left(s_{1}^{*}, s_{2}^{*}, s_{3}^{*}, u_{1}^{*}, u_{2}^{*}, u_{3}^{*}, v_{1}^{*}, v_{2}^{*}, v_{3}^{*}, \ell_{1}^{*}, \ell_{2}^{*}, \ell_{3}^{*}\right)$ be a rest point of $(2.2)$ such that $2-f_{u}\left(s_{i}^{*}\right)>0,2-f_{v}\left(s_{i}^{*}\right)>0$ and $2-f_{\ell}\left(s_{i}^{*}\right)>0, i=1,2,3$.

(i) $u_{1}^{*}>0$ if and only if $u_{2}^{*}>0$ and $u_{3}^{*}>0$

$v_{1}^{*}>0$ if and only if $v_{2}^{*}>0$ and $v_{3}^{*}>0$

$\ell_{1}^{*}>0$ if and only if $\ell_{2}^{*}>0$ and $\ell_{3}^{*}>0$

$$
s_{1}^{*}>s_{2}^{*}>s_{3}^{*}>0
$$

(ii) $f_{u}\left(s_{1}^{*}\right)>f_{u}\left(s_{2}^{*}\right)>f_{u}\left(s_{3}^{*}\right)$

$f_{v}\left(s_{1}^{*}\right)>f_{v}\left(s_{2}^{*}\right)>f_{v}\left(s_{3}^{*}\right)$

$f_{\ell}\left(s_{1}^{*}\right)>f_{\ell}\left(s_{2}^{*}\right)>f_{\ell}\left(s_{3}^{*}\right)$

(iii) If $u_{1}^{*}>0$,

$$
\left(2-f_{u}\left(s_{1}^{*}\right)\right)\left(2-f_{u}\left(s_{2}^{*}\right)\right)\left(2-f_{u}\left(s_{3}^{*}\right)\right)+f_{u}\left(s_{1}^{*}\right)+f_{u}\left(s_{3}^{*}\right)=4
$$

(iv) If $v_{1}^{*}>0$,

$$
\left(2-f_{v}\left(s_{1}^{*}\right)\right)\left(2-f_{v}\left(s_{2}^{*}\right)\right)\left(2-f_{v}\left(s_{3}^{*}\right)\right)+f_{v}\left(s_{1}^{*}\right)+f_{v}\left(s_{3}^{*}\right)=4 \text {. }
$$

(v) If $\ell_{1}^{*}>0$,

$$
\left(2-f_{\ell}\left(s_{1}^{*}\right)\right)\left(2-f_{\ell}\left(s_{2}^{*}\right)\right)\left(2-f_{\ell}\left(s_{3}^{*}\right)\right)+f_{\ell}\left(s_{1}^{*}\right)+f_{\ell}\left(s_{3}^{*}\right)=4 .
$$

Proof. Since the statements are quite, we point out the basics for $u_{i}^{*}$-the facts for $v_{i}^{*}$ and $\ell_{i}^{*}$ follow similarly. The first three statements in (i) follow directly from the equations for $u_{i}^{*}, v_{i}^{*}$ and $\ell_{i}^{*}$. The third equation in (2.2) at equilibrium yields:

$$
s_{2}^{*}-2 s_{3}^{*}=f_{u}\left(s_{3}^{*}\right) u_{3}^{*}+f_{v}\left(s_{3}^{*}\right) v_{3}^{*}+f_{\ell}\left(s_{3}^{*}\right) \ell_{3}^{*} \geq 0
$$


then

$$
s_{2}^{*} \geq 2 s_{3}^{*} \geq s_{3}^{*} .
$$

Similarly, the second equation in (2.2) at equilibrium yields:

$$
s_{1}^{*}-2 s_{2}^{*}+s_{3}^{*}=f_{u}\left(s_{2}^{*}\right) u_{2}^{*}+f_{v}\left(s_{2}^{*}\right) v_{2}^{*}+f_{\ell}\left(s_{2}^{*}\right) \ell_{2}^{*} \geq 0
$$

hence,

$$
s_{1}^{*} \geq 2 s_{2}^{*}-s_{3}^{*}>2 s_{2}^{*}-s_{2}^{*}=s_{2}^{*}
$$

From (3.1) and (3.2), we get:

$$
s_{1}^{*}>s_{2}^{*}>s_{3}^{*}
$$

Direct substitution into the equations in (2.2) represents that $s_{3}^{*}$ cannot be zero. Since $f_{u}$ is monotone increasing (ii) follows.

To prove (iii), from equations (2.2), notice that the following homogeneous equations:

$$
\begin{aligned}
& u_{2}^{*}-\left(2-f_{u}\left(s_{1}^{*}\right)\right) u_{1}^{*}=0 \\
& u_{1}^{*}+u_{3}^{*}-\left(2-f_{u}\left(s_{2}^{*}\right)\right) u_{2}^{*}=0 \\
& u_{2}^{*}-\left(2-f_{u}\left(s_{3}^{*}\right)\right) u_{3}^{*}=0
\end{aligned}
$$

are linear in $u_{1}^{*}, u_{2}^{*}, u_{3}^{*}$. If $u_{i}^{*}>0, i=1,2,3$; the determinant of the coefficient matrix of equations (3.4) must equal to zero. From (i), it follows that:

$$
\left(2-f_{u}\left(s_{1}^{*}\right)\right)\left(2-f_{u}\left(s_{2}^{*}\right)\right)\left(2-f_{u}\left(s_{3}^{*}\right)\right)+f_{u}\left(s_{1}^{*}\right)+f_{u}\left(s_{3}^{*}\right)=4
$$

This completes the proof of the lemma.

It is clear that there is a critical point of the form:

$$
\left(\frac{3}{4}, \frac{1}{2}, \frac{1}{4}, 0,0,0,0,0,0\right)
$$

Also, there exist some rest points (in sight of Lemma 3.1(i)) of the form

$$
\begin{gathered}
\left(\hat{s}_{1}, \hat{s}_{2}, \hat{s}_{3}, \hat{u}_{1}, \hat{u}_{2}, \hat{u}_{3}, 0,0,0,0,0,0\right), \\
\left(\tilde{s}_{1}, \tilde{s}_{2}, \tilde{s}_{3}, 0,0,0, \tilde{v}_{1}, \tilde{v}_{2}, \tilde{v}_{3}, 0,0,0\right)
\end{gathered}
$$

and

$$
\left(\check{s}_{1}, \check{s}_{2}, \check{s}_{3}, 0,0,0,0,0,0, \check{\ell}_{1}, \check{\ell}_{2}, \check{\ell}_{3}\right)
$$

with all nonzero positive components. Each of these corresponds to the case of one organism in the gradostat. The existence of such points and their (global) stability has been studied analytically for $n$ vessels by Tang [12]. But those statements are conditioned in terms of the parameter $D$ which we have scaled out. We will obtain their existence on geometric grounds. 


\section{The System with a Single Species}

To analyze the system (2.4) it is necessary to study the flow on the boundary, hence we begin to investigate the rest points with one population. Such analysis can be found in Tang [12] but with $n$, vessels. In the case of three vessels, we will use simple geometric arguments which will be useful later.

If one of the competitors is absent the system (2.4) takes the form (e.g. set $v_{i}=$ $\left.0, \ell_{i}=0, i=1,2,3\right)$ :

$$
\begin{aligned}
& u_{1}^{\prime}=-2 u_{1}+u_{2}+f_{u}\left(\frac{3}{4}-u_{1}\right) u_{1}, \\
& u_{2}^{\prime}=u_{1}-2 u_{2}+u_{3}+f_{u}\left(\frac{1}{2}-u_{2}\right) u_{2}, \\
& u_{3}^{\prime}=u_{2}-2 u_{3}+f_{u}\left(\frac{1}{4}-u_{3}\right) u_{3} .
\end{aligned}
$$

Define

$$
\alpha_{i}(z)=2-f_{u}\left(\left(1-\frac{i}{4}\right)-z\right), i=1,2,3 .
$$

Let us assume that the system (4.1) has at least one simple steady state $E$ : $\left(\hat{u}_{1}, \hat{u}_{2}, \hat{u}_{3}\right)$ in the interior of the first octant, i.e. the equations:

$$
\begin{aligned}
& u_{2}-\alpha_{1}\left(u_{1}\right) u_{1}=0 \\
& u_{1}+u_{3}-\alpha_{2}\left(u_{2}\right) u_{2}=0 \\
& u_{2}-\alpha_{3}\left(u_{3}\right) u_{3}=0
\end{aligned}
$$

have at least one simple solution $\left(\hat{u}_{1}, \hat{u}_{2}, \hat{u}_{3}\right)$ with $\hat{u}_{i}>0, i=1,2,3$. This equivalent to the assumption that the determinant of the variational matrix, $J_{u}$ of $(4.1)$ at $\left(\hat{u}_{1}, \hat{u}_{2}, \hat{u}_{3}\right)$ is not zero, i.e.,

$$
\begin{gathered}
\operatorname{det} J_{u_{b}=\beta_{1}}+\beta_{3}-\left[\alpha_{1} \alpha_{2} \beta_{3}+\alpha_{1} \alpha_{3} \beta_{2}+\alpha_{2} \alpha_{3} \beta_{1}+\alpha_{1} \beta_{2} \beta_{3}\right. \\
\left.+\alpha_{2} \beta_{1} \beta_{3}+\alpha_{3} \beta_{1} \beta_{2}+\beta_{1} \beta_{2} \beta_{3}\right] \neq 0
\end{gathered}
$$

where

$$
J_{u}\left(\hat{u}_{1}, \hat{u}_{2}, \hat{u}_{3}\right)=\left(\begin{array}{ccc}
-\left(\alpha_{1}+\beta_{1}\right) & 1 & 0 \\
1 & -\left(\alpha_{2}+\beta_{2}\right) & 1 \\
0 & 1 & -\left(\alpha_{3}+\beta_{3}\right)
\end{array}\right)
$$

and $\alpha_{i}^{\prime} s$ are defined in (4.2) with $z=\hat{u}_{i}, i=1,2,3$,

$$
\begin{aligned}
& \beta_{1}=\beta_{1}\left(\hat{u}_{1}\right)=\frac{m_{u} a_{u} \hat{u}_{1}}{\left(a_{u}+\frac{3}{4}-\hat{u}_{1}\right)^{2}}, \\
& \beta_{2}=\beta_{2}\left(\hat{u}_{2}\right)=\frac{m_{u} a_{u} \hat{u}_{2}}{\left(a_{u}+\frac{1}{2}-\hat{u}_{2}\right)^{2}}, \\
& \beta_{3}=\beta_{3}\left(\hat{u}_{3}\right)=\frac{m_{u} a_{u} \hat{u}_{3}}{\left(a_{u}+\frac{1}{4}-\hat{u}_{3}\right)^{2}} .
\end{aligned}
$$


The characteristic equation is:

$$
\lambda^{3}+P_{1}(\hat{u}) \lambda^{2}+P_{2}(\hat{u}) \lambda+P_{3}(\hat{u})=0
$$

where

$$
\begin{aligned}
P_{1}(\hat{u})= & \alpha_{1}+\alpha_{2}+\alpha_{3}+\beta_{1}+\beta_{2}+\beta_{3} \\
P_{2}(\hat{u})= & \left(\alpha_{2}+\beta_{2}\right)\left(\alpha_{3}+\beta_{3}\right)+\left(\alpha_{1}+\beta_{1}\right)\left(\alpha_{2}+\alpha_{3}+\beta_{2}+\beta_{3}\right)-2 \\
P_{3}(\hat{u})= & \alpha_{1} \alpha_{2} \beta_{3}+\alpha_{1} \alpha_{3} \beta_{2}+\alpha_{2} \alpha_{3} \beta_{1}+\alpha_{1} \beta_{2} \beta_{3} \\
& \quad+\alpha_{2} \beta_{1} \beta_{3}+\alpha_{3} \beta_{1} \beta_{2}+\beta_{1} \beta_{2} \beta_{3}-\beta_{1}-\beta_{3}
\end{aligned}
$$

The assumption (4.3) implies that the equation (4.5) has no zero root.

An equilibrium of a differential system is called hyperbolic, see Hirsch and Smale [5, p.305] if its characteristic equation has no roots with zero real parts (the roots may be distinct or repeated). The linearization technique about a hyperbolic point determines the asymptotic behaviour of solutions near that point. Therefore, we assume that the roots $\lambda_{i}$ of (4.5) are not purely imaginary.

The nature of the roots of the cubic equation (4.5) depends on the values

$$
\begin{aligned}
M(\hat{u}) & =\frac{1}{27}\left(P_{2}(\hat{u})-\frac{1}{3} P_{1}^{2}(\hat{u})\right)^{3}+\frac{1}{4}\left(\frac{2}{27} P_{1}^{3}(\hat{u})-\frac{1}{3} P_{1}(\hat{u}) P_{2}(\hat{u})+P_{3}(\hat{u})\right)^{2} \\
& =\frac{1}{27} Q^{3}(\hat{u})+\frac{1}{4}\left(R(\hat{u})-\frac{P_{1}(\hat{u})}{3} Q(\hat{u})\right)^{2}, \\
H(\hat{u}) & =P_{1}(\hat{u}) P_{2}(\hat{u})-P_{3}(\hat{u}), \\
Q(\hat{u}) & =P_{2}(\hat{u})-\frac{1}{3} P_{1}^{2}(\hat{u}), \\
R(\hat{u}) & =P_{3}(\hat{u})-\frac{1}{27}
\end{aligned}
$$

where $P_{1}(\hat{u}), P_{2}(\hat{u})$ and $P_{3}(\hat{u})$ are given by (4.6) (see for example Reyn [10]).

Three distinct real roots of $(4.5)$ occur when $M(\hat{u})<0$, one real and two complex roots when $M(\hat{u})>0$ and at least two coincidental roots when $M(\hat{u})=0$. The various signs of $M(\hat{u})$ lead to various nature and stability (or instability) properties of the corresponding equilibrium $E$ according to $H(\hat{u})>0$ (or $H(\hat{u})<0$ ) (see [1]).

We will investigate the stability of the rest point $E:\left(\hat{u}_{1}, \hat{u}_{2}, \hat{u}_{3}\right)$ in $R_{+}^{n} \cap \Gamma$ where $n=3$ in this section and $n=9$ later.

Lemma 4.1 The critical point $E:\left(\hat{u}_{1}, \hat{u}_{2}, \hat{u}_{3}\right)$ with $\hat{u}_{i}>0, i=1,2,3$ is asymptotically stable if

$$
\alpha_{1}\left(\hat{u}_{1}\right) \alpha_{2}\left(\hat{u}_{2}\right) \geq 2 \text { and } \alpha_{2}\left(\hat{u}_{2}\right) \alpha_{3}\left(\hat{u}_{3}\right) \geq 1
$$


Proof. A set of necessary and sufficient conditions for all the roots of the cubic equation (4.5) to have negative real parts is, by the Routh-Hurwitz criterion,

$$
P_{1}(\hat{u})>0, P_{3}(\hat{u})>0 \text { and } H(\hat{u})>0
$$

where $H(\hat{u})=P_{1}(\hat{u}) P_{2}(\hat{u})-P_{3}(\hat{u})$ and $P_{i}(\hat{u})$ 's are defined in (4.6). Since $\alpha_{i}\left(\hat{u}_{i}\right)>0, i=$ $1,2,3$ (bj Lemma 3.1) and $\beta_{i}\left(\hat{u}_{i}\right), i=1,2,3$ are positive, then

$$
P_{1}(\hat{u})>0
$$

A rearrangement of the third equation in (4.6), yields

$$
\begin{gathered}
P_{3}(\hat{u})=\alpha_{1} \alpha_{3} \beta_{2}+\alpha_{1} \beta_{2} \beta_{3}+\alpha_{2} \beta_{1} \beta_{3}+\alpha_{3} \beta_{1} \beta_{2}+\beta_{1} \beta_{2} \beta_{3} \\
+\beta_{3}\left(\alpha_{1} \alpha_{2}-1\right)+\beta_{1}\left(\alpha_{2} \alpha_{3}-1\right)
\end{gathered}
$$

It is clear that the right-hand side of (4.10) is positive if $\alpha_{1} \alpha_{2} \geq 1$ and $\alpha_{2} \alpha_{3} \geq 1$.

$$
\begin{aligned}
H(\hat{u})= & \alpha_{1} \alpha_{2} \alpha_{3}+2 \beta_{1} \beta_{2} \beta_{3}+2 \alpha_{3}\left(\alpha_{1}+\alpha_{2}\right)\left(\beta_{1}+\beta_{2}+\beta_{3}\right) \\
& +\left(\alpha_{1}^{2}+\beta_{1}^{2}\right)\left(\alpha_{2}+\alpha_{3}+\beta_{2}+\beta_{3}\right) \\
& +\left(\alpha_{2}^{2}+\beta_{2}^{2}\right)\left(\alpha_{3}+\beta_{1}+\beta_{3}\right)+\alpha_{1} \beta_{2}^{2} \\
& +\left(\alpha_{3}^{2}+\beta_{3}^{2}\right)\left(\alpha_{1}+\alpha_{2}+\beta_{1}+\beta_{2}\right) \\
& +\alpha_{2}\left(\alpha_{1} \alpha_{2}-2\right)+\beta_{1}\left(2 \alpha_{1} \alpha_{2}-1\right) \\
& +2 \beta_{2}\left(\alpha_{1} \alpha_{2}-1\right)+\beta_{3}\left(2 \alpha_{1} \alpha_{2}-1\right)
\end{aligned}
$$

Then $H(\hat{u})>0$ if $\alpha_{1} \alpha_{2} \geq 2$ since $\beta_{i}$ 's and $\alpha_{i}$ 's are positive. Hence, from (4.9), (4.10) and (4.11) that the criterion (4.8) holds if $\alpha_{1} \alpha_{2} \geq 2$ and $\alpha_{2} \alpha_{3} \geq 1$ are satisfied. This completes the proof of the Lemma.

Remark 4.1 The investigation of the conditions under which the equilibrium point $E:\left(\hat{u}_{1}, \hat{u}_{2}, \hat{u}_{3}\right), \hat{u}_{i}^{\prime}$ 's $>0$ is unstable using the Routh-Hurwitz criterion (4.8) is impossible since (4.8) fails if and only if $\alpha_{i}\left(\hat{u}_{i}\right)<0$ which contradicts with lemma 3.1.

The stability (or instability) conditions of the origin $(0,0,0)$ can be found by using the Routh-Hurwitz criterion as follows:

The variational matrix $J_{u}$ has $\beta_{i}=0, i=1,2,3$ at the origin so

$$
J_{u}(0,0,0)=\left(\begin{array}{ccc}
-\alpha_{1}(0) & 1 & 0 \\
1 & -\sigma_{2}(0) & 1 \\
0 & 1 & -\alpha_{3}(0)
\end{array}\right)
$$

The characteristic equation is

$$
\lambda^{3}+P_{1}(0) \lambda^{2}+P_{2}(0) \lambda+P_{3}(0)=0
$$

where

$$
\begin{aligned}
& P_{1}(0)=\alpha_{1}(0)+\alpha_{2}(0)+\alpha_{3}(0) \\
& P_{2}(0)=\alpha_{1}(0) \alpha_{2}(0)+\alpha_{1}(0) \alpha_{3}(0)+\alpha_{2}(0) \alpha_{3}(0)-2, \\
& P_{3}(0)=\alpha_{1}(0) \alpha_{2}(0) \alpha_{3}(0)-\alpha_{1}(0)-\alpha_{3}(0)
\end{aligned}
$$

And $P_{3}(0) \neq 0$. 
Lemma 4.2 The origin $(0,0,0)$ is asymptotically stable if

$$
\alpha_{i}(0)>1, \quad i=1,2,3 \text {. }
$$

Proof. As in the proof of Lemma 4.1, the origin is asymptotically stable if and only if, by using the Routh-Hurwitz criterion,

$$
P_{1}(0)>0, P_{3}(0)>0 \text { and } H(0)>0
$$

where $H(0)=P_{1}(0) P_{2}(0)-P_{3}(0)$ and $P_{i}(0)$ 's are defined in (4.13).

If $\alpha_{i}(0)$ 's $>1, P_{1}(0)>0$ automatically hold and

$$
\alpha_{1}(0) \alpha_{2}(0) \alpha_{3}(0)>\alpha_{1}(0)+\alpha_{3}(0) .
$$

Moreover, $H(0)>0$ if $\alpha_{i}(0)$ 's $>1$, since

$$
\begin{aligned}
H(0)= & \alpha_{1}^{2}(0) \alpha_{3}(0)+\alpha_{2}^{2}(0)\left(\alpha_{1}(0) \alpha_{3}(0)\right)+\alpha_{3}^{2}(0) \alpha_{2}(0) \\
& +\alpha_{1}(0)\left(\alpha_{1}(0) \alpha_{2}(0)-1\right)+\alpha_{3}(0)\left(\alpha_{3}(0) \alpha_{1}(0)-1\right) \\
& +2 \alpha_{2}(0)\left(\alpha_{1}(0) \alpha_{3}(0)-1\right) .
\end{aligned}
$$

This completes the proof of the lemma.

Lemma 4.3 The origin $(0,0,0)$ is unstable if

(i) $0<\alpha_{i}(0) \leq 1$ or

(ii) $\alpha_{i}(0)<0, \quad i=1,2,3$.

Proof.

(i) It is clear that $P_{3}(0)<0$, if $0<\alpha_{i}(0) \leq 1$

(ii) It is obvious that $P_{1}(0)<0$ if $\alpha_{i}(0)<0$.

In each of the cases (i) and (ii) the Routh-Hurwitz criterion (4.15) fails then the origin $(0,0,0)$ is unstable. This completes the proof of the lemma.

Lemma 4.4. The critical point $E:\left(\hat{u}_{1}, \hat{u}_{2}, \hat{u}_{3}\right), \hat{u}_{i}$ 's $>0$ is the only nontrivial critical point in the first octant.

Proof. Suppose there were two distinct rest point $\left(\hat{u}_{1}, \hat{u}_{2}, \hat{u}_{3}\right),\left(\tilde{u}_{1}, \tilde{u}_{2}, \tilde{u}_{3}\right)$ with

$$
\hat{u}_{i}>0, \quad \tilde{u}_{i}>0, \quad i=1,2,3 .
$$

Assume that the labelling is such that $\hat{u}_{3} \leq \tilde{u}_{3}$. Then, it follows that:

$$
\hat{u}_{2}=\alpha_{3}\left(\hat{u}_{3}\right) \hat{u}_{3} \leq \alpha_{3}\left(\tilde{u}_{3}\right) \tilde{u}_{3}=\tilde{u}_{2} \text {. }
$$

The equality holds if $\hat{u}_{3}=\tilde{u}_{3}$. Then, if $\left(\hat{u}_{1}, \hat{u}_{2}, \hat{u}_{3}\right) \neq\left(\tilde{u}_{1}, \tilde{u}_{2}, \tilde{u}_{3}\right)$ it follows that:

$$
\begin{aligned}
0 & =\alpha_{1}\left(\hat{u}_{1}\right) \alpha_{2}\left(\hat{u}_{2}\right) \alpha_{3}\left(\hat{u}_{3}\right)-\alpha_{1}\left(\hat{u}_{1}\right)-\alpha_{3}\left(\hat{u}_{3}\right) \\
& \neq \alpha_{1}\left(\tilde{u}_{1}\right) \alpha_{2}\left(\tilde{u}_{2}\right) \alpha_{3}\left(\tilde{u}_{3}\right)-\alpha_{1}\left(\tilde{u}_{1}\right)-\alpha_{3}\left(\tilde{u}_{3}\right) \\
& =0
\end{aligned}
$$

a contradiction. Thus, there is only one critical point in the interior of the positive cone. 
Lemma 4.5 If the origin $(0,0,0)$ is unstable (i.e. $\alpha_{i}(0)<0$ or $0<\alpha_{i}(0) \leq$ $1, i=1,2,3)$ and if $a_{u}+\left(1-\frac{i}{4}\right)<2 \hat{u}_{i}, a_{u}>1$ then the nontrivial equilibrium point $E:\left(\hat{u}_{1}, \hat{u}_{2}, \hat{u}_{3}\right)$ is asymptotically siable.

Proof. From (4.2),

$$
\begin{aligned}
\alpha_{i}(0) & =2-\frac{\left(1-\frac{i}{4}\right) m_{u}}{a_{u}+\left(1-\frac{i}{4}\right)}, \\
\alpha_{i}\left(\hat{u}_{i}\right) & =2-\frac{m_{u}\left[\left(1-\frac{i}{4}\right)-\hat{u}_{i}\right]}{a_{u}+\left(1-\frac{i}{4}\right)-\hat{u}_{i}}, \quad i=1,2,3
\end{aligned}
$$

If $\alpha_{i}(0)<0$, from $(4.15)$ we obtain

$$
m_{u}>\frac{2 a_{u}}{1-\frac{i}{4}}+2, \quad i=1,2,3
$$

Using the inequality (4.17) and $a_{u}+\left(1-\frac{i}{4}\right)<2 \hat{u}_{i}, a_{u}>1$, in (4.16), we have

$$
\begin{aligned}
\alpha_{i}\left(u_{i}\right) & >\frac{2 a_{u}+2\left(1-\frac{i}{4}\right)-2 \hat{u}_{i}+2\left(\frac{a_{u}}{1-(i / 4)}+1\right)\left(\hat{u}_{i}-(1-(i / 4))\right)}{\hat{u}_{i}} \\
& =\frac{2 a_{u}}{1-(i / 4)} \\
& >1
\end{aligned}
$$

since $1-(i / 4)<1, i=1,2,3$. Hence, the condition (4.7) is satisfied which establishes the stability of the rest point $E:\left(\hat{u}_{1}, \hat{u}_{2}, \hat{u}_{3}\right)$.

If $\alpha_{i}(0) \leq 1$, from $(4.15)$ we get:

$$
m_{u} \geq \frac{a_{u}}{1-(i / 4)}+1
$$

Using (4.18) and $a_{u}+(1-(i / 4))<2 \hat{u}_{i}, a_{u}>1$, in (4.16) we have

$$
\alpha_{i}\left(\hat{u}_{i}\right)>\frac{a_{u}-(1-(i / 4))}{(1-(i / 4))}+\frac{a_{u}+(1-(i / 4))}{\hat{u}_{i}}>1
$$

since $1-(i / 4)<1, i=1,2,3$. Therefore, the condition (4.7) is satisfied which proves the stability of steady state $E:\left(\hat{u}_{1}, \hat{u}_{2}, \hat{u}_{3}\right)$. This completes the proof of the lemma.

Remark 4.2 Like lemmas apply to the equations for the $v$ population,

$$
\begin{aligned}
& v_{1}^{\prime}=-2 v_{1}+v_{2}+f_{v}\left(\frac{3}{4}-v_{1}\right) v_{1} \\
& v_{2}^{\prime}=v_{1}-2 v_{2}+v_{3}+f_{v}\left(\frac{1}{2}-v_{2}\right) v_{2} \\
& v_{3}^{\prime}=v_{2}-2 v_{3}+f_{v}\left(\frac{1}{4}-v_{3}\right) v_{3}
\end{aligned}
$$


with $\alpha_{i+3}(z)=2-f_{v}(1-(i / 4)-z)$, replacing $\alpha_{i}(z)$ in $(4.2), i=1,2,3$.

And also for $\ell$ population,

$$
\begin{aligned}
& \ell_{1}^{\prime}=-2 \ell_{1}+\ell_{2}+f_{\ell}\left(\frac{3}{4}-\ell_{1}\right) \ell_{1} \\
& \ell_{2}^{\prime}=\ell_{1}-2 \ell_{2}+\ell_{3}+f_{\ell}\left(\frac{1}{2}-\ell_{2}\right) \ell_{2} \\
& \ell_{3}^{\prime}=\ell_{2}-2 \ell_{3}+f_{\ell}\left(\frac{1}{4}-\ell_{3}\right) \ell_{3}
\end{aligned}
$$

wiht $\alpha_{i+6}(z)=2-f_{\ell}\left(\left(1-\frac{i}{4}\right)-z\right)$ replacing $\alpha_{i}(z)$ in $(4.2), i=1,2,3$.

\section{The Stability of the Critical Points}

In this section, we will investigate the stability of the critical points for the system (2.4). The arguments are based on standard variational methods but the size of the variational matrix, $9 \times 9$, makes the computation not easy. The variational matrix for (2.4) is:

$$
J=\left(\begin{array}{lll}
A_{1} & C_{1} & C_{1} \\
C_{2} & A_{2} & C_{2} \\
C_{3} & C_{3} & A_{3}
\end{array}\right)
$$

where each of $A_{i}$ and $C_{i}, i=1,2,3$ is $3 \times 3$ matrix,

$$
\begin{aligned}
& A_{i}=\left(\begin{array}{ccc}
-\left(\alpha_{3 i-2}+\gamma_{3 i-2}\right) & 1 & 0 \\
1 & -\left(\alpha_{3 i-1}+\gamma_{3 i-1}\right) & 1 \\
0 & 1 & -\left(\alpha_{3 i}+\gamma_{3 i}\right)
\end{array}\right) \\
& C_{i}=\left(\begin{array}{ccc}
-\gamma_{3 i-2} & 0 & 0 \\
0 & -\gamma_{3 i-1} & 0 \\
0 & 0 & -\gamma_{3 i}
\end{array}\right) ; i=1,2,3
\end{aligned}
$$

$\alpha_{j}$ 's and $\beta_{j}$ 's are given by :

$$
\begin{aligned}
\alpha_{i} & =2-f_{u}\left(\left(1-\frac{i}{4}\right)-u_{i}-v_{i}-\ell_{i}\right), \\
\alpha_{i+3} & =2-f_{v}\left(\left(1-\frac{i}{4}\right)-u_{i}-v_{i}-\ell_{i}\right), \\
\alpha_{i+6} & =2-f_{\ell}\left(\left(1-\frac{i}{4}\right)-u_{i}-v_{i}-\ell_{i}\right), \\
\gamma_{i} & =\frac{a_{u} m_{u} u_{i}}{\left(a_{u}+\left(1-\frac{i}{4}\right)-u_{i}-v_{i}-\ell_{i}\right)^{2}}, \\
\gamma_{i+3} & =\frac{a_{v} m_{v} v_{i}}{\left(a_{v}+\left(1-\frac{i}{4}\right)-u_{i}-v_{i}-\ell_{i}\right)^{2}}, \\
\gamma_{i+6} & =\frac{a_{\ell} m_{\ell} \ell_{i}}{\left(a_{\ell}+\left(1-\frac{i}{4}\right)-u_{i}-v_{i}-\ell_{i}\right)^{2}}, i=1,2,3 .
\end{aligned}
$$


The $\alpha_{i}$ 's are the same quantities used in Section $4, z=u_{i}+v_{i}+\ell_{i}, i=1,2,3$, if $J$ is evaluated at a point

$$
\left(u_{1}, u_{2}, u_{3}, v_{1}, v_{2}, v_{3}, \ell_{1}, \ell_{2}, \ell_{3}\right)
$$

and are positive if

$$
\left(u_{1}, \ddot{u}_{2}, u_{3}, v_{1}, v_{2}, v_{3}, \ell_{1}, \ell_{2}, \ell_{3}\right)
$$

is a rest point (Lemma 3.1).

Let $\Sigma$ denote the rest point set of the system (2.4) in $\Gamma$. Let $E^{0}$ denote the origin;

$$
\begin{gathered}
\hat{E},\left(\hat{u}_{1}, \hat{u}_{2}, \hat{u}_{3}, 0,0,0,0,0,0\right), \hat{u}_{i} \text { 's }>0 ; \\
\tilde{E},\left(0,0,0, \tilde{v}_{1}, \tilde{v}_{2}, \tilde{v}_{3}, 0,0,0\right), \tilde{v}_{i} \text { 's }>0, \\
\check{E},\left(0,0,0,0,0,0, \check{\ell}_{1}, \check{\ell}_{2}, \check{\ell}_{3}\right), \tilde{\ell}_{i}^{\prime} s>0 ; \\
E^{\star},\left(u_{1}^{\star}, u_{2}^{\star}, u_{3}^{\star}, v_{1}^{\star}, v_{2}^{\star}, v_{3}^{\star}, \ell_{1}^{\star}, \ell_{2}^{\star}, \ell_{3}^{\star}\right), \\
u_{i}^{\star}, v_{i}^{\star}, \ell_{i}^{\star}>0, i=1,2,3 . E^{0}
\end{gathered}
$$

always exists, that is the case of all ecological systems. In Section 4 , we have seen conditions for the existence of $\hat{E}, \tilde{E}$. In this section, we give a (weak) necessary condition for $E^{*}$ to exist.

Lemma 5.1 $\Sigma=\left\{E^{0}\right\}$ if and only if

$$
\alpha_{i}(0)>1, \quad i=1, \ldots, 9
$$

i.e. all trajectories with initial conditions in $\Gamma$ tend to $E^{0}$.

Proof. From (5.4) and (5.3), the matrices $C_{i}, i=1,2,3$ are zero matrices at $E^{0}$, so the variational matrix $J$, evaluated at $E^{0}$ is:

$$
J_{E^{0}}=\left(\begin{array}{ccc}
J_{u}(0,0,0) & 0 & 0 \\
0 & J_{v}(0,0,0) & 0 \\
0 & 0 & J_{\ell}(0,0,0)
\end{array}\right)
$$

where $J_{u}(0,0,0), J_{v}(0,0,0)$, and $J_{\ell}(0,0,0)$ are as in Section 4. Hence, in view of Lemma 4.2 , (5.5) makes $E^{0}$ local asymptotic stable.

We will use a comparison argument to prove that $E^{0}$ is global asymptotic stable. Suppose $u(t)=\left(u_{1}(t), u_{2}(t), u_{3}(t), v_{1}(t), v_{2}(t), v_{3}(t), \ell_{1}(t), \ell_{2}(t), \ell_{3}(t)\right)$ be a solution of (2.4), $u_{i}(0), v_{i}(0), \ell_{i}(0)>0, i=1,2,3$; and let $z(t)=\left(z_{1}(t), z_{2}(t), z_{3}(t)\right)$ be a solution of $(4.1)$ with $z_{i}(0)=u_{i}(0), i=1,2,3$. Since $f_{u}\left(\left(1-\frac{i}{4}\right)-\eta\right), i=1,2,3$ are monotone decreasing functions in $\eta, u_{i}, \ell_{i}, v_{i}>0$, the first three components of $u(t)$ satisfy:

$$
\begin{aligned}
& u_{1}^{\prime}(t)<u_{2}(t)-\left(2-f_{u}\left(\frac{3}{4}-u_{1}(t)\right)\right) u_{1}(t), \\
& u_{2}^{\prime}(t)<u_{1}(t)+u_{3}(t)-\left(2-f_{u}\left(\frac{1}{2}-u_{2}(t)\right)\right) u_{2}(t), \\
& u_{3}^{\prime}(t)<u_{2}(t)-\left(2-f_{u}\left(\frac{1}{4}-u_{3}(t)\right)\right) u_{3}(t),
\end{aligned}
$$


while the components of $z(t)$ satisfy an equation with the same right hand side. Hence, for all $t>0$ (see [2], Theorem 10, Chap. 1), $u_{i}(t) \leq z_{i}(t)$ and since $z_{i}(t) \rightarrow 0$ so $u_{i}(t) \rightarrow 0, i=1,2,3$. A similar argument represents that $v_{i}(t) \rightarrow 0, \ell_{i}(t) \rightarrow 0, i=1,2,3$. Therefore, all solutions in the positive cone tend to $E^{0}$. This completes the proof of the Lemma.

\section{Remark 5.1}

1. If $\alpha_{i}(0)<0$ or $0<\alpha_{i}(0) \leq 1$, $a_{u}+\left(1-\frac{i}{4}\right)<2 \hat{u}_{i}, a_{u}>1, \quad i=1,2,3$, and $\alpha_{j+3}(0)>1, j=1, \ldots, 6$, then

$$
\Sigma=\left\{E^{0}, \hat{E}\right\} .
$$

2. If $\alpha_{i+3}(0)<0$ or $0<\alpha_{i+3}(0) \leq 1$,

$a_{v}+\left(1-\frac{i}{4}\right)<2 \tilde{v}_{i}, a_{v}>1$,

$\alpha_{i}(0)>1$ and $\alpha_{i+6}(0)>1, i=1,2,3$, then

$$
\Sigma=\left\{E^{0}, \tilde{E}\right\} .
$$

3. If $\alpha_{i+6}(0)<0$ or $0<\alpha_{i+6}(0) \leq 1$,

$a_{\ell}+\left(1-\frac{i}{4}\right)<2 \check{\ell}_{i}, a_{\ell}>1, i=1,2,3$,

and $\alpha_{j}(0)>1, j=1, \ldots, 6$; then

$$
\Sigma=\left\{E^{0}, \check{E}\right\}
$$

Moreover, by using the above comparison argument we can devote a (weak) necessary condition for $E^{\star}$ to exist.

Remark 5.2 A necessary condition for $E^{\star}$ to exist is that $\hat{E}, \tilde{E}$ and $\check{E}$ exist simultaneously.

\section{Lemma 3.2}

(i) The critical point $E\left(\hat{u}_{1}, \hat{u}_{2}, \hat{u}_{3}, 0,0,0,0,0,0\right)$ is locally asymptotically stable if

$$
\alpha_{i}\left(\hat{u}_{i}\right)>1, \quad i=1,2,3 .
$$

(ii) The equilibrium point $\tilde{E}\left(0,0,0, \tilde{v}_{1}, \tilde{v}_{2}, \tilde{v}_{3}, 0,0,0\right)$ is locally asymptotically stable if

$$
\alpha_{i}\left(\tilde{v}_{i}\right)>1, \quad i=1,2,3 .
$$

(iii) The steady state $\check{E}\left(0,0,0,0,0,0, \check{\ell}_{1}, \check{\ell}_{2}, \check{\ell}_{3}\right)$ is locally asymptotically stable if

$$
\alpha_{i}\left(\check{\ell}_{i}\right)>1, \quad i=1,2,3 .
$$

Proof. We will prove the statement (i) - the statements (ii) and (iii) follow similarly. At $\hat{E}, \gamma_{j}=0, j=4,5, \ldots, 9$; and $\gamma_{i}=\beta_{i}, i=1,2,3 ; \beta_{i}$ 's are defined in the previous 
section. A sufficient condition for $J$, evaluated at $\hat{E}$, to have eigenvalues with negative real parts is that the off diagonal elements are replaced by their absolute values, the determinants of the principal minors alternate in sign [11]. Let $d_{i}, i=1, \ldots, 9$ denote to the principal minors of

$$
\left(\begin{array}{ccc}
\hat{A}_{1} & \hat{C}_{1} & \hat{C}_{1} \\
0 & \hat{A}_{2} & 0 \\
0 & 0 & \hat{A}_{3}
\end{array}\right)
$$

where $\hat{C}_{1}$ is a diagonal matrix and $\hat{A}_{i}$ 's are 3 -squared matrices

$$
\begin{aligned}
& \hat{C}_{1}=\left(\begin{array}{lll}
\beta_{1} & & \\
& \beta_{2} & \\
& & \beta_{3}
\end{array}\right), \\
& \hat{A}_{1}=\left(\begin{array}{ccc}
-\left(\alpha_{1}+\beta_{1}\right) & 1 & 0 \\
1 & -\left(\alpha_{2}+\beta_{2}\right) & 1 \\
0 & 1 & -\left(\alpha_{3}+\beta_{3}\right)
\end{array}\right),
\end{aligned}
$$

and

$$
\hat{A}_{k}=\left(\begin{array}{ccc}
-\alpha_{3 k-2} & 1 & 0 \\
1 & -\alpha_{3 k-1} & 1 \\
0 & 1 & -\alpha_{3 k}
\end{array}\right), k=2,3
$$

In fact,

$$
\begin{aligned}
d_{1} & =-\left(\alpha_{1}+\beta_{1}\right)<0 \text { and } \\
d_{2} & =\left(\alpha_{1}+\beta_{1}\right)\left(\alpha_{2}+\beta_{2}\right)-1 \\
& >0
\end{aligned}
$$

since $\alpha_{1}>1, \alpha_{2}>1$. Moreover

$$
\begin{aligned}
d_{3}= & -\alpha_{1} \alpha_{3} \beta_{2}-\alpha_{1} \beta_{2} \beta_{3}-\alpha_{3} \beta_{1} \beta_{2}-\left(\alpha_{1} \alpha_{2}-1\right) \beta_{3} \\
& \quad-\left(\alpha_{2} \alpha_{3}-1\right) \beta_{1}-\beta_{1} \beta_{2} \beta_{3} \\
< & 0
\end{aligned}
$$

since $\alpha_{1} \alpha_{2} \alpha_{3}-\alpha_{1}-\alpha_{3}=0$ by Lemma 3.1. Also $d_{4}=-\alpha_{4} d_{3}>0$ because $d_{3}<0$. Too, $d_{5}=\left(\alpha_{4} \alpha_{5}-1\right) d_{3}<0$, since $\alpha_{4} \alpha_{5}>1$ and $d_{3}<0$.

$$
d_{6}=-\left(\alpha_{4} \alpha_{5} \alpha_{6}-\alpha_{4}-\alpha_{6}\right) d_{3}>0
$$

since $\alpha_{5}^{\prime}$ s $>1, \alpha_{4} \alpha_{5} \alpha_{6}>\alpha_{4}+\alpha_{6}$.

$$
\begin{aligned}
d_{7} & =-\alpha_{7} d_{6} \\
& <0 \quad\left(\text { since } d_{6}>0\right) \\
d_{8} & =-\alpha_{8} d_{7}-d_{6} \\
& =\left(\alpha_{7} \alpha_{8}-1\right) d_{6} \\
& >0
\end{aligned}
$$


because $\alpha_{7} \alpha_{8}>1$ and $d_{6}>0$. Finally,

$$
\begin{aligned}
d_{9} & =-\alpha_{9} d_{8}-d_{7} \\
& =-\left(\alpha_{7} \alpha_{8} \alpha_{9}-\alpha_{7}-\alpha_{8}\right) d_{6} \\
& <0
\end{aligned}
$$

since $\alpha_{7} \alpha_{8} \alpha_{9}>\alpha_{7}+\alpha_{8}$ and $d_{6}>0$. This completes the proof of the lemma.

The size of the variational matrix $J$, makes the investigation of the local stability of the rest point $E^{\star}\left(u_{1}^{\star}, u_{2}^{\star}, u_{3}^{\star}, v_{1}^{\star}, v_{2}^{\star}, v_{3}^{\star}, \ell_{1}^{\star}, \ell_{2}^{\star}, \ell_{3}^{\star}\right), u_{i}^{\star}$ 's $>0, v_{i}^{\star}$ 's $>0,, \ell_{i}^{\star}$ 's $>0$, since $\gamma_{j} \neq 0, j=1,2, \ldots, 9$, evaluated at $E^{\star}$, causes difficulty in the computation of the determinants of the principal minors of

$$
\left(\begin{array}{lll}
A_{1}^{\star} & C_{1}^{\star} & C_{1}^{\star} \\
C_{2}^{\star} & A_{2}^{\star} & C_{2}^{\star} \\
C_{3}^{\star} & C_{3}^{\star \star} & A_{3}^{\star}
\end{array}\right)
$$

where $A_{i}^{*}=A_{i}, A_{i}$ 's are defined in (5.2),

$$
C_{i}^{*}=\left(\begin{array}{ccc}
\gamma_{3 i-2} & 0 & 0 \\
0 & \gamma_{3 i-1} & 0 \\
0 & 0 & \gamma_{3 i}
\end{array}\right)
$$

$i=1,2,3 ; \alpha_{i}$ 's and $\gamma_{i}$ 's are evaluated at $E^{\star}$. Although we proved the alternation of the sign until the fifth principal minors of (5.6) under the condition $\alpha_{i}^{\prime}$ 's $>1$ Let $d_{i}, i=$ $1, \ldots, 9$ denote to the principal minors of (5.6). In fact

$$
\begin{aligned}
d_{1}= & -\left(\alpha_{1}+\gamma_{1}\right) \\
< & 0 \\
d_{2}= & \alpha_{1} \alpha_{2}+\alpha_{1} \gamma_{2}+\alpha_{2} \gamma_{1}+\gamma_{1} \gamma_{2}-1 \\
> & 0 \quad\left(\text { since } \alpha_{3}^{\prime} s>1\right) \\
d_{3}= & -\left[\alpha_{1} \alpha_{3} \gamma_{2}+\alpha_{1} \gamma_{2} \gamma_{3}+\alpha_{2} \gamma_{1} \gamma_{3}+\alpha_{3} \gamma_{1} \gamma_{2}\right. \\
& \left.\quad+\left(\alpha_{1} \alpha_{2}-1\right) \gamma_{3}+\left(\alpha_{2} \alpha_{3}-1\right) \gamma_{1}+\gamma_{1} \gamma_{2} \gamma_{3}\right] \\
< & 0 \quad \alpha_{1} \alpha_{3} \alpha_{4} \gamma_{2}+\alpha_{1} \alpha_{3} \gamma_{2} \gamma_{4}+\alpha_{1} \alpha_{4} \gamma_{1} \gamma_{3}+\alpha_{1} \gamma_{2} \gamma_{3} \gamma_{4} \\
& \quad+\alpha_{2} \alpha_{4} \gamma_{1} \gamma_{3}+\alpha_{3} \alpha_{4} \gamma_{1} \gamma_{2}+\left(\alpha_{1} \alpha_{2}-1\right)\left(\alpha_{4}+\gamma_{4}\right) \gamma_{3} \\
& \quad+\left(\alpha_{2} \alpha_{3}-1\right) \alpha_{4} \gamma_{1} \\
>0 & \quad-\left(\alpha_{1} \alpha_{3}-1\right) \alpha_{5} \gamma_{2} \gamma_{4}-\left(\alpha_{1} \alpha_{5}-1\right)\left(\alpha_{3}+\gamma_{3}\right) \gamma_{2} \gamma_{4} \\
d_{5}= & \left(\alpha_{4} \alpha_{5}-1\right) d_{3}-\left(\alpha_{1} \alpha_{2}-1\right)\left(\alpha_{4} \gamma_{5}+\alpha_{5} \gamma_{4}\right) \gamma_{3} \\
& \quad-\left(\alpha_{2} \alpha_{4}-1\right) \gamma_{1} \gamma_{3} \gamma_{5}-\left(\alpha_{3}-1\right)\left(\alpha_{1}+\alpha_{1} \alpha_{4}+\alpha_{4} \gamma_{1}\right) \gamma_{2} \gamma_{5} \\
& \quad-\left(\alpha_{2} \alpha_{3} \alpha_{4}-\alpha_{3}-\alpha_{4}\right) \gamma_{1} \gamma_{5} \\
<0 &
\end{aligned}
$$

since $\alpha_{i}$ 's $>1$ and $d_{3}<0$. 


\section{References}

[1] Bojadziev, G. and Sattar, M.A., "Perturbations in the Three Dimensional Kolmogorov Model," Math. Biosci. 78: 293-305 (1986).

[2] Coppel, W.A., Stability and Asymptotic Behaviour of Differential Equaitons, Boston: Heath 1965.

[3] Hansin, S.R. and Hubbel, S.P., "Single Nutrient Microbial Competitors Argument between Experimental and Theoretical Forecast Outcomes, Science 20, 1491-1493 (1980).

[4] Herbert, D., Elsworth, R. and Telling, R.C., "The Continuous Culture of Bacteria: a Theoretical and Experimental Study, J. Gen. Microbiol. 14, 601-622.

[5] Hirsch, M.W. and Smale, S., Differential Equations, Dynamical Systems, and Linear Algebra, Academic, New York, 1974.

[6] Hsu, S.B., "Limiting Behaviour for Competing Species, SIAM J. Appl. Math. 34, 760-763 (1978).

[7] Hsu, S.B., Hubbel, S.P. and Waltman, P., "A Mathematical Theory for Single Nutrient in Continuous Cultures of Microorganisms," SIAM J. Appl. Math. 32, 366-383 (1977).

[8] Jager, W., Joseph, W.;H. So, Tang, B. and Waltman, p., "Competition in the Gradostat," J. Math. Biol. 25: 23-42 (1987).

[9] Lovitt, R.W. and Wimpenny, J.W.T., "The Gradostat: a Bidirectional Compound Chemostat and Its Applications in Microbiological Research," Gen. Microbiol. 127, 261-268 (1981).

[10] Reyn, J.W., "Classification and Description of Singular Points of a System of Three Linear Differential Equations," Z. Angew Math. Phys. 15: 540-557 (1964).

[11] Smith, H., "Systems of Ordinary Differential Equations which Generate and Order Preserving Flow: A Survey of Results," SIAM Rev. (to appear).

[12] Tang, B., "Mathematical Investigation of Microorganisms in the Gradostat," J. Math. Biol. 23, 319-339 (1986).

[13] Taylor, P.W. and Williams, J.L., "Theoretical Studies on the Coexistence of Competing Species under Continuous Flow Conditions," Can. J. Microbiol. 21, 90-98 (1975).

[14] Waltman, P., Hubbel, S.P. and Hsu, S.P., "Theoretical and Experimental Investigation of Microbial Competition in Continuous Culture." In: Burton, T. (Ed.) Modeling and Differential Equations in Biology, New York: Marcel Dekker 1980.

[15] Wimpenny, J.W.T. and Lovitt, R.W., "The Investigation and Analysis of Heterogeneous Environments Using the Gradostat." In: Grainger, J.M., Lynch, J.M. (Ed.), Microbiological Methods for Environmental Biotechnology, Orlando: Academic Press 1984.

Department of Mathematics, Faculty of Science, Al-Azhar University, Nasr City, Cairo, EGYPT.

Department of Mathematics, Faculty of Science, Beni-Sweif, EGYPT. 